\title{
POWER SERIES WHOSE SECTIONS HAVE ZEROS OF LARGE MODULUS
}

\author{
BY \\ J. D. BUCKHOLTZ( $\left.{ }^{1}\right)$
}

1. Introduction. Several theorems in the theory of polynomials deal with the problem of obtaining bounds for the modulus of one or more zeros of a polynomial, $a_{0}+a_{1} z+\cdots+a_{n} z^{n}$, when certain of the coefficients $a_{0}, a_{1}, \ldots, a_{k}$, are regarded as fixed, and the remaining are arbitrary (cf. [2, Chapter 8]). In the present paper we apply results of this nature to partial sums of a power series $\sum a_{p} z^{p}$. For each positive integer $n, \mathfrak{r}_{n}$ will denote the radius of the largest circle with center at $z=0$ whose interior contains no zero of the $n$th section,

$$
s_{n}(z)=\sum_{p=0}^{n} a_{p} z^{p} .
$$

We shall be concerned primarily with growth properties of the sequence $\left\{\mathfrak{r}_{n}\right\}$. The most interesting case is that in which $\sum a_{p} z^{p}$ is the power series for an entire function which omits the value zero. It is not hard to show that this is equivalent to having $\lim \mathfrak{r}_{n}=\infty$. One can, however, construct other power series for which $\lim \sup \mathrm{r}_{n}=\infty$.

Since nothing is lost by doing so, we shall always suppose that $a_{0}=1$. This assumption will be used freely and without explicit mention. For notational convenience, $\Sigma$ and $\Sigma^{\prime}$ will denote sums taken over the nonnegative and positive integers, respectively.

In §2, upper bounds for $\mathfrak{r}_{n}$ are obtained from algebraic relations between the zeros of $s_{n}(z)$ and the first "few" of the numbers $a_{1}, a_{2}, a_{3}, \cdots$. From algebraic considerations alone, we show that

$$
1+\mathfrak{r}_{n}=n^{o(1)}
$$

except possibly for certain "exceptional" power series, and, further, that these exceptions must be power series for entire functions of the form $\exp \{P(z)\}$, where $P(z)$ is a polynomial.

In $\S 3$ we use analytic methods to obtain lower bounds for $\mathfrak{r}_{n}$ in case $\sum a_{p} z^{p}$ is an entire function which omits the value zero. We are able to show that the "apparent exceptions" to (1.1) are actual exceptions, and thus characterize entire functions of the form $\exp \{P(z)\}$ for $P(z)$ a polynomial.

Presented to the Society, January 24, 1964; received by the editors May 3, 1963 and, in revised form, October 30, 1963.

(1) Sponsored by the United States Army under contract no. DA-11-022-ORD-2059. 
Taken together, the upper and lower bounds yield a number of asymptotic properties of the sequence $\left\{\mathfrak{r}_{n}\right\}$. For $\sum a_{p} z^{p}=\exp \{g(z)\}$, where $g(z)$ is an entire function of order $\rho$, we show that

$$
\limsup \frac{\log \log n}{\log \mathfrak{r}_{n}}=\rho .
$$

This and similar results are discussed in $\$ 4$.

In $\$ 5$ we prove that

$$
\lim \sup \mathfrak{r}_{n}\left|a_{n}\right|^{1 / n}=1,
$$

provided that $\sum a_{p} z^{p}$ is an entire function of infinite order which has no zeros. Making use of (1.2) and the observation that $\left|a_{n}\right|^{-1 / n}$ is the geometric mean of the zeros of $s_{n}(z)$, we deduce the following: If $\sum a_{p} z^{p}$ is an entire function of infinite order without zeros, $\varepsilon>0$, and $\varepsilon^{\prime}>0$, then, for infinitely many integers $n$, fewer than $n \varepsilon^{\prime}$ zeros of $s_{n}(z)$ have moduli greater than $\mathfrak{r}_{n}(1+\varepsilon)$. This result is of some interest in connection with theorems of F. Carlson [1] and P. Rosenbloom [6] on zeros of sections of entire series of infinite order.

2. Upper bounds. Let $\Sigma^{\prime} b_{p} z^{p}$ be the power series obtained formally from the identity

$$
\frac{\sum p a_{p} z^{p-1}}{\sum a_{p} z^{p}}=\sum^{\prime} p b_{p} z^{p-1}
$$

THEOREM 2.1. If $k$ is a positive integer such that $b_{k} \neq 0$, and $n \geqq k$, then $s_{n}(z)$ has a zero in the disc

Proof. If one lets

$$
|z| \leqq\left\{\frac{n}{k\left|b_{k}\right|}\right\}^{1 / k}
$$

$$
\frac{s_{n}^{\prime}(z)}{s_{n}(z)}=\Sigma^{\prime} p b_{p}^{(n)} z^{p-1},
$$

and observes that $b_{p}^{(n)}=b_{p}$ for $p \leqq n$, the result then follows from a theorem of G. Sz.-Nagy ([3], [4], and [2, Example 2, p. 43]).

As a consequence of the above, we have

$$
\mathfrak{r}_{n}=O\left(n^{1 / k}\right)
$$

for every value of $k$ for which $b_{k} \neq 0$. If $\Sigma a_{p} z^{p}=\exp \{P(z)\}$ for some polynomial $P(z)$, then (2.1) holds with $k$ equal to the degree of $P(z)$. If $\sum a_{p} z^{p}$ is not a power series of this form, then (2.1) holds for infinitely many integers $k$. This establishes (1.1).

Corollary 2.2 .

$$
\liminf \frac{\log \mathfrak{r}_{n}}{\log \log n} \leqq \liminf \frac{\log \left(1 /\left|b_{k}\right|\right)}{k \log k}
$$


Proof. From Theorem 2.1 we have

$$
\frac{\log \mathfrak{r}_{n}}{\log k} \leqq \frac{\log n}{k \log k}-\frac{1}{k}+\frac{\log \left(1 /\left|b_{k}\right|\right)}{k \log k}
$$

Choose $n=n(k)$ so that $\log n \sim k$, and let $k \rightarrow \infty$.

The above result is of particular interest if $\Sigma^{\prime} b_{p} z^{p}=g(z)$, where $g(z)$ is an entire function of order $\rho$. We then have $\Sigma a_{p} z^{p}=\exp \{g(z)\}$, and

$$
\lim \sup \frac{\log \log n}{\log \mathfrak{r}_{n}} \geqq \rho,
$$

since the right-hand side of (2.2) is $1 / \rho$. Later we shall see that equality holds in (2.3).

For certain entire functions $g(z)$, Theorem 2.1 can be used to obtain an extremely good upper bound for $\mathfrak{r}_{n}$. For this purpose we make use of the maximum term function, $\mu(r)=\mu(r, g)$, defined by

$$
\mu(r)=\max _{p}\left\{\left|b_{p}\right| r^{p}\right\},
$$

and the central index, $v(r)$, which is the largest integer $m$ such that

$$
\mu(r)=\left|b_{m}\right| r^{m} \text {. }
$$

THEOREM 2.3. Let $\Sigma a_{p} z^{p}=\exp \{g(z)\}$, where $g(z)=\Sigma^{\prime} b_{p} z^{p}$ is an entire function of finite order. For each $n$, let $\beta_{n}$ be the positive number such that $\mu\left(\beta_{n}\right)=n$, where $\mu(r)$ is defined by (2.4). Then for all sufficiently large $n, s_{n}(z)$ has a zero in the disc $|z| \leqq \beta_{n}$.

Proof. From Theorem 2.1,

$$
\mathfrak{r}_{n} \leqq\left\{\frac{n}{k\left|b_{k}\right|}\right\}^{1 / k} \leqq\left\{\frac{n}{\left|b_{k}\right|}\right\}^{1 / k}
$$

Let $k=v\left(\beta_{n}\right)$. Then $\left|b_{k}\right| \beta_{n}^{k}=\mu\left(\beta_{n}\right)=n$. Therefore $\mathfrak{r}_{n} \leqq \beta_{n}$.

It remains to show that $k \leqq n$, or equivalently, that $v\left(\beta_{n}\right) \leqq \mu\left(\beta_{n}\right)$. This is true provided the inequality

$$
v(r)<\mu(r)
$$

holds for all sufficiently large $r$. A proof of (2.5) follows easily from the relation $[10$, p. 34]

$$
\limsup \frac{\log v(r)}{\log r}=\rho,
$$

where $\rho$ is the order of $g(z)$. The hypothesis that $\rho$ is finite can, therefore, be replaced by (2.5).

3. Lower bounds. We obtain lower bounds for the numbers $\mathfrak{r}_{n}$ under the assumption that $\sum a_{p} z^{p}=\exp \{g(z)\}$, where $g(z)=\Sigma^{\prime} b_{p} z^{p}$ is an entire function. 
$G(z)$ will denote the majorant of $g(z)$ defined by

$$
G(z)=\Sigma^{\prime}\left|b_{p}\right| z^{p} .
$$

We note for future use that the order of $G(z)$ is the same as that of $g(z)$; we shall also need the inequality

$$
\Sigma\left|a_{p}\right| r^{p} \leqq \exp \{G(r)\} \quad \text { if } r \geqq 0 .
$$

A proof of (3.2) follows from expanding $\exp \{G(r)\}$ as a power series in $r$ and observing that the coefficient of $r^{p}$ is at least as great as $\left|a_{p}\right|$.

THeOREM 3.1. Let $\sum a_{p} z^{p}=\exp \{g(z)\}$, where $g(z)$ is an entire function with majorant $G(z)$ given by (3.1). If $n$ is a positive integer, then

$$
\mathfrak{r}_{n}>r \exp \{-2 G(r) / n\} \quad \text { for all } r \geqq 0 .
$$

In particular, if $\alpha_{n}$ is the positive number such that $G\left(\alpha_{n}\right)=n$, then

$$
\mathfrak{r}_{n}>\alpha_{n} / e^{2} \text {. }
$$

Furthermore, if $g(z)$ is not a polynomial, then for $\varepsilon>0$, one has

$$
\mathfrak{r}_{n}>\alpha_{n}(1-\varepsilon)
$$

for all sufficiently large $n$.

Proof. Suppose $r>0$ and let $f(z)=\sum a_{p} z^{p}$. We shall establish (3.3) by showing that

$$
|z| \leqq r \exp \{-2 G(r) / n\}
$$

implies

$$
\left|1-s_{n}(z) / f(z)\right|<1,
$$

and therefore that $s_{n}(z) \neq 0$. The latter is obviously true if $z=0$; suppose $z$ satisfies (3.6) and $z \neq 0$. Then $0<|z|<r$, and $|1 / f(z)|=|\exp \{-g(z)\}|<\exp \{G(r)\}$. Also,

$$
\begin{aligned}
\left|f(z)-s_{n}(z)\right| & \leqq\left.\left.|z| r\right|^{n} \sum_{p=n+1}^{\infty}\left|a_{p}\right| r^{p}|z| r\right|^{p-n} \\
& <\left.|z| r\right|^{n} \sum_{p=n+1}^{\infty}\left|a_{p}\right| r^{p} \\
& <\left.|z| r\right|^{n} \exp \{G(r)\}
\end{aligned}
$$

by virtue of (3.2). Therefore

$$
\left|1-s_{n}(z) / f(z)\right|<\{|z| r \mid \exp \{2 G(r) / n\}\}^{n} \leqq 1,
$$

by (3.6). This proves (3.3). If $r=\alpha_{n}$, we have (3.4). 
The proof of (3.5) depends on the following property of $G(r)$ (cf. [5, Vol. 2, p. 4]): If $g(z)$ is not a polynomial, and $0<c<1$, then

$$
\lim _{r \rightarrow \infty} \frac{G(c r)}{G(r)}=0 .
$$

We now make use of (3.7) and the sequence $\left\{\alpha_{n}\right\}$ to construct a sequence $\left\{c_{n}\right\}$ such that

$$
\lim c_{n}=1 \text { and } \lim \frac{G\left(c_{n} \alpha_{n}\right)}{G\left(\alpha_{n}\right)}=0
$$

In (3.3) let $r=c_{n} \alpha_{n}$ and replace $n$ by $G\left(\alpha_{n}\right)$. Then

$$
\mathfrak{r}_{n}>c_{n} \alpha_{n} \exp \left\{-2 G\left(c_{n} \alpha_{n}\right) / G\left(\alpha_{n}\right)\right\} \text {. }
$$

Observing that

establishes (3.5).

$$
\lim c_{n} \exp \left\{-2 G\left(c_{n} \alpha_{n}\right) / G\left(\alpha_{n}\right)\right\}=1
$$

4. Asymptotic properties. In a number of cases, fairly precise information about the sequence $\left\{\mathfrak{r}_{n}\right\}$ can be obtained by comparing the upper bounds of $\$ 2$ with the lower bounds developed in $\$ 3$.

TheOREM 4.1. If $\sum a_{p} z^{p}=\exp \{P(z)\}$, where $P(z)$ is a polynomial of degree $k$, then there are positive numbers $A$ and $B$ such that

$$
A n^{1 / k}<\mathfrak{r}_{n}<B n^{1 / k}, \quad n=1,2,3, \cdots .
$$

The proof, which is omitted, follows easily from (2.1) and (3.4).

In view of (1.1), one sees that Theorem 4.1 characterizes power series for entire functions of the form $\exp \{P(z)\}$. Among all power series, the exponential series (more accurately, the series for $a e^{b z}$ ) is the only one for which $\mathfrak{r}_{n}$ increases as rapidly as a linear function of $n$. Zeros of sections and remainders of this series have been investigated by G. Szegö [8].

Our next theorem is similar in some respects to a theorem of $\mathbf{M}$. Tsuji [9] on the maximum modulus of zeros of sections of an entire series. If we let $\boldsymbol{R}_{\boldsymbol{n}}$ denote the largest modulus of a zero of $s_{n}(z)$ (with the convention that $R_{n}=\infty$ if $a_{n}=0$ ), Tsuji's theorem asserts that

$$
\limsup \frac{\log n}{\log R_{n}}
$$

is equal to the order of $\sum a_{p} z^{p}$. For an entire function $\sum a_{p} z^{p}$ which omits zero we obtain an analogous result involving $\mathfrak{r}_{n}$ and the order of the logarithm of $\sum a_{p} z^{p}$.

THEOREM 4.2. If $\sum a_{p} z^{p}=\exp \{g(z)\}$, where $g(z)$ is an entire function of order $\rho(0 \leqq \rho \leqq \infty)$, then 


$$
\limsup \frac{\log \log n}{\log \mathfrak{x}_{n}}=\rho \text {. }
$$

Proof. Since the order of $G(z)$ is also $\rho$, we have

$$
\begin{aligned}
\rho & =\underset{r \rightarrow \infty}{\limsup } \frac{\log \log G(r)}{\log r} \geqq \limsup _{n \rightarrow \infty} \frac{\log \log n}{\log \alpha_{n}} \\
& \geqq \limsup \frac{\log \log n}{\log \left(\mathfrak{x}_{n} e^{2}\right)}
\end{aligned}
$$

by (3.4). Since $\log \left(\mathfrak{r}_{n} e^{2}\right) \sim \log \mathfrak{r}_{n}$, we can neglect the factor $e^{2}$. The other half of the proof follows from (2.3).

If $g(z)$ is of finite positive order $\rho$ and of type $\tau(0 \leqq \tau \leqq \infty)$, one can prove a sharper result, namely, that

$$
\limsup \frac{\log n}{\mathfrak{r}_{n}^{\rho}}=\tau .
$$

For this one needs (3.5) in place of (3.4); the " $\geqq$ " half of the result is obtained from Theorem 2.1 by a procedure similar to the proof of Corollary 2.2. In this case one chooses $n=n(k)$ so that $\log n \sim k / \rho$.

If $g(z)$ is of finite order, the asymptotic relation [5, Vol. 2, p. 8]

$$
\log G(r) \sim \log \mu(r, G)=\log \mu(r, g)
$$

yields information about the relative sizes of $\alpha_{n}$ and $\beta_{n}$. From (4.1) and the definitions of $\alpha_{n}$ and $\beta_{n}$, we have

$$
\log G\left(\alpha_{n}\right) \sim \log G\left(\beta_{n}\right)
$$

This supplies a connecting link between our upper and lower bounds for $\mathfrak{r}_{n}$.

THEOREM 4.3. If $\sum a_{p} z^{p}=\exp \{g(z)\}$, where $g(z)$ is an entire function of finite order, then

$$
\log \alpha_{n} \sim \log \mathfrak{r}_{n} \sim \log \beta_{n} .
$$

Proof. Since $\alpha_{n} / e^{2}<\mathfrak{r}_{n} \leqq \beta_{n}$ for large $n$, it suffices to prove that $\log \alpha_{n} \sim \log \beta_{n}$. If we note that $\alpha_{n} \leqq \beta_{n}$, the result follows from (4.2) and the Hadamard three circle theorem (applied to circles with radii $1, \alpha_{n}$, and $\beta_{n}$ ).

THEOREM 4.4. Let $\sum a_{p} z^{p}=\exp \{g(z)\}$, where $g(z)$ is an entire function of finite order. If, for some $\delta>0$, there is a nondecreasing function $H(r)$ such that $\log G(r) \sim r^{\delta} H(r)$, then

$$
\alpha_{n} \sim \mathfrak{r}_{n} \sim \beta_{n}
$$


Proof. Suppose $\varepsilon>0$. The condition on $G(r)$ implies that $g(z)$ is not a polynomial; therefore $\alpha_{n}(1-\varepsilon)<\mathfrak{r}_{n} \leqq \beta_{n}$ for large $n$, and we need only prove that $\alpha_{n} \sim \beta_{n}$.

From (4.2) and the condition on $G(r)$, we have

$$
1 \geqq\left[\frac{\alpha_{n}}{\beta_{n}}\right]^{\delta} \sim \frac{H\left(\beta_{n}\right)}{H\left(\alpha_{n}\right)} \geqq 1,
$$

since $\alpha_{n} \leqq \beta_{n}$ and $H(r)$ is nondecreasing. Hence $\alpha_{n} \sim \beta_{n}$.

As a special case of the above, we note that the condition $\log G(r) \sim \tau r^{p}$ for positive numbers $\rho$ and $\tau$ implies that

$$
\mathfrak{r}_{n} \sim\left[\frac{\log n}{\tau}\right]^{1 / \rho}
$$

For $g(z)$ of finite order, $\log G(r) \sim \log M_{g}(r)$, where $M_{g}(r)$ is the maximum modulus of $g(z)$ on $|z|=r$. Therefore the condition on $G(r)$ in the hypothesis of Theorem 4.4 is equivalent to the corresponding condition on $M_{g}(r)$. In addition, we note that Theorems 4.3 and 4.4 remain valid if $\alpha_{n}$ is replaced by $\alpha_{n}^{\prime}$, where $\alpha_{n}^{\prime}$ is defined by $M_{g}\left(\alpha_{n}^{\prime}\right)=n$.

5. Comparison with the coefficients. In this section we restrict our attention to the case $\sum a_{p} z^{p}=\exp \{g(z)\}$, where $g(z)$ is an entire function which is not a polynomial. This is equivalent to requiring that $\Sigma a_{p} z^{p}$ be an entire function of infinite order without zeros. We shall compare the lower bound (3.5) for $\mathfrak{r}_{n}$ with the elementary upper bound

$$
\mathfrak{r}_{n} \leqq\left|a_{n}\right|^{-1 / n} \quad \text { if } a_{n} \neq 0 .
$$

(The right-hand side of (5.1) is the geometric mean of the moduli of zeros of $s_{n}(z)$.) Our principal result is the following:

THEOREM 5.1. If $\sum a_{p} z^{p}$ is an entire function of infinite order without zeros, then

$$
\limsup \alpha_{n}\left|a_{n}\right|^{1 / n}=\limsup \mathfrak{r}_{n}\left|a_{n}\right|^{1 / n}=1 .
$$

Before proving Theorem 5.1 we shall consider two of its corollaries.

CoRollary 5.2. Let $\sum a_{p} z^{p}$ satisfy the hypothesis of Theorem 5.1. If $\varepsilon>0$, then

$$
\alpha_{n}(1-\varepsilon)<\mathfrak{r}_{n} \text { and } \mathfrak{r}_{n}\left|a_{n}\right|^{1 / n} \leqq 1
$$

for all sufficiently large $n$, and

$$
\alpha_{n}(1+\varepsilon)>\mathfrak{r}_{n}>(1-\varepsilon)\left|a_{n}\right|^{-1 / n}
$$

for infinitely many $n$. 
Proof. Theorem 5.1 and inequalities (3.5) and (5.1).

COROLlARY 5.3. If $\sum a_{p} z^{p}$ is an entire function of infinite order without zeros, $\varepsilon>0$ and $\varepsilon^{\prime}>0$, then for infinitely many integers $n$, fewer than $n \varepsilon^{\prime}$ zeros of $s_{n}(z)$ have moduli greater than $\mathfrak{r}_{n}(1+\varepsilon)$.

Proof. Choose $\delta$ so that

$$
0<\delta<1-(1+\varepsilon)^{-\varepsilon^{\prime}}
$$

If $n$ is a positive integer for which $\mathfrak{r}_{n}>(1-\delta)\left|a_{n}\right|^{-1 / n}$, then an easy calculation shows that fewer than $n \varepsilon^{\prime}$ zeros of $s_{n}(z)$ have moduli greater than $\mathfrak{r}_{n}(1+\varepsilon)$.

Proof of Theorem 5.1. From (3.5) and (5.1) it follows that

$$
\limsup \alpha_{n}\left|a_{n}\right|^{1 / n} \leqq \limsup \mathfrak{r}_{n}\left|a_{n}\right|^{1 / n} \leqq 1 ;
$$

consequently, we have only to prove that

$$
\limsup \alpha_{n}\left|a_{n}\right|^{1 / n} \geqq 1 \text {. }
$$

To facilitate the proof of (5.2) we first establish two lemmas. These will enable us to obtain a lower bound for $\alpha_{n}$ in terms of the maximum modulus of $\Sigma a_{p} z^{p}$.

LEMMA 5.1a. For all $r>0$,

$$
\alpha_{n}>r \exp \{-2 G(r) / n\} \text {. }
$$

Proof. The function $u(r)=r \exp \{-2 G(r)\}$ assumes its maximum at the number $r=\gamma_{n}$ such that $2 \gamma_{n} G^{\prime}\left(\gamma_{n}\right)=n$. Since $2 r G^{\prime}(r)>G(r)$ for all $r>0$, we have

$$
\alpha_{n}>\gamma_{n}>\gamma_{n} \exp \left\{-2 G\left(\gamma_{n}\right) / n\right\} .
$$

LEMMA 5.1b. If $\varepsilon>0$, then

$$
\alpha_{n}>\frac{r}{1+\varepsilon}[M(r)]^{-8 / n \varepsilon} \quad \text { for all } r>0,
$$

where

$$
M(r)=\max _{|z|=r}\left|\sum a_{p} z^{p}\right|
$$

Proof. The proof depends on the following variant of the Borel-Carathéodory inequality (proved, but not explicitly stated, in [10, pp. 17-20]): If $0<r<R$, then

$$
G(r)=\Sigma^{\prime}\left|b_{p}\right| r^{p} \leqq \frac{4 r}{R-r} A(R)
$$

where

$$
A(R)=\max _{|z|=R}\left\{\operatorname{Re} \Sigma^{\prime} b_{p} z^{p}\right\}
$$


If we rewrite (5.3) in the form

$$
\alpha_{n}>\frac{r}{1+\varepsilon} \exp \left\{-\frac{2}{n} G\left(\frac{r}{1+\varepsilon}\right)\right\}
$$

and in (5.5) replace $r$ and $R$ by $r /(1+\varepsilon)$ and $r$ respectively, we have

$$
\alpha_{n}>\frac{r}{1+\varepsilon} \exp \left\{\frac{-8 A(r)}{n \varepsilon}\right\} \text {. }
$$

Since $A(r)=\log M(r)$, the result follows.

It is worth noting that the inequality of Lemma $5.1 \mathrm{~b}$ remains valid if $\alpha_{n}$ is replaced by $\mathfrak{r}_{n}$. To see this one uses (3.3) in place of (5.3).

We are now in a position to establish (5.2). Let $\mu(r)$ and $v(r)$ denote (contrary to previous usage) the maximum term and central index of the series $\sum a_{p} z^{p}$. If we choose $n=v(r)$, the inequality of Lemma $5.1 \mathrm{~b}$ can be written as

$$
\log \left\{(1+\varepsilon) \alpha_{n}\left|a_{n}\right|^{1 / n}\right\}>\frac{\log \mu(r)}{v(r)}-\frac{8}{\varepsilon} \frac{\log M(r)}{v(r)} .
$$

Since $\sum a_{p} z^{p}$ is of infinite order, it follows that

$$
\liminf \frac{\log M(r)}{v(r)}=0
$$

from a theorem of S. M. Shah [7]. Therefore

$$
\lim \sup (1+\varepsilon) \alpha_{n}\left|a_{n}\right|^{1 / n} \geqq 1,
$$

which establishes (5.2) and completes the proof.

Theorem 5.1 adds an interesting footnote to certain more general results on zeros of sections of power series. If $\sum a_{p} z^{p}$ is an entire function of infinite order and $\varepsilon>0$, it is known [1], [6] that, for infinitely many integers $n$, all but $o(n)$ zeros of $s_{n}(z)$ lie in the annulus

$$
(1-\varepsilon)\left|a_{n}\right|^{-1 / n}<|z|<(1+\varepsilon)\left|a_{n}\right|^{-1 / n} \text {. }
$$

If $\sum a_{p} z^{p}$ omits the value zero, we have shown that, for infinitely many integers $n$, no zero of $s_{n}(z)$ lies in the interior of the inner circle of the annulus.

\section{REFERENCES}

1. F. Carlson, Sur les fonctions entières, Ark. Mat. Astronom. Fys. 35A (1948), no. 14, 1-18.

2. M. Marden, The geometry of the zeros of a polynomial in a complex variable, Math. Surveys No. 3, Amer. Math. Soc., Providence, R. I., 1949.

3. G. Sz.-Nagy, Ueber einen Satz von Laguerre, J. Reine Angew. Math. 169 (1933), 186-192.

4. - Die Polarkreise eines Punktes in bezug auf ein Polynom, Portugal. Math. 7 (1948), 51-57. 
5. G. Pólya and G. Szegö, Aufgaben und Lehrsätze aus der Analysis, Vols. 1, 2, 2nd ed., Springer, Berlin, 1954.

6. P. C. Rosenbloom, Distribution of zeros of polynomials, Lectures on functions of a complex variable, pp. 265-285, Univ. of Michigan Press, Ann Arbor, Mich., 1955.

7. S. M. Shah, The maximum term of an entire series. IV, Quart. J. Math. Oxford Ser. (2) 1 (1950), 112-116.

8. G. Szegö, Über eine Eigenschaft der Exponentialreihe, S.-B. Berlin Math. Ges. 23 (1924), $50-64$.

9. M. Tsuji, On the distribution of zero points of sections of a power series. III, Japan. J. Math. 3 (1926), 49-52.

10. G. Valiron, Lectures on the general theory of integral functions, Privat, Toulouse, 1923.

UNIVERSITY OF WISCONSIN,

Madison, Wisconsin

University of North Carolina,

Chapel hill, North Carolina 